\title{
Distal common bile duct adenoma
}

Khanin Khanungwanitkul, M.D. ${ }^{(1)}$

Tanawat Pattarapuntakul, M.D. ${ }^{(2)}$

Naruemon Wisedopas, M.D. ${ }^{(3)}$

From ${ }^{(1)}$ Department of Radiology, Faculty of Medicine, Prince of Songkla University, Songkhla, Thailand.

${ }^{(2)}$ Department of Internal Medicine, Faculty of Medicine, Prince of Songkla University, Songkhla, Thailand.

(3) Department of Pathology, Faculty of Medicine, Chulalongkorn University, Bangkok, Thailand.

Address correspondence to K.K. (e-mail: khanin14@gmail.com)

Keywords: Common bile duct adenoma, Benign tumor, Bile duct, Imaging findings, Clinical presentation.

\section{Introduction}

Adenoma is a benign tumor usually found in the gastrointestinal tract $[1,2]$. However, it is rarely found in the extrahepatic bile duct. Although it is a benign tumor, it may cause an aggressive clinical course like a malignant disease which is more common[2]. Furthermore, there is still limited understanding of its nature, imaging finding and management of the disease. We reported a case of distal common bile duct adenoma which includes its clinical course, imaging finding and pathologic result. 


\section{case summary}

An 86-year-old man presented with intermittent abdominal pain for 2 months. He had an underlying malignant gastric stromal tumor with hepatic metastasis diagnosed for 7 years, having been treated with Imatinib. The physical examination reveals low-grade fever, no pale or icteric sclera, a normal liver span, a normal active bowel sound, and no ascites. A complete blood count shows mild anemia, a normal white blood count and platelets. The liver function test shows slightly elevated serum alanine transaminase and aspartate aminotransferase of 289 and $815 \mathrm{U} / \mathrm{L}$, respectively.

\section{Imaging, endoscopic, and pathologic findings}

Magnetic Resonance Imaging (MRI) with a hepatocyte specific contrast agent was performed (Figure 1). The study shows gastric mass and multiple cystic lesions in the liver which are decreased in size from the prior study (not shown). The diagnosis of these lesions reveals an improvement of his underlying gastrointestinal stromal tumor (GIST). Furthermore, the MRI also shows diffused dilatation of the bilateral IHDs and CBD with a round-shaped filling defect in the distal CBD. Regardless of its enhancement, the interpreted radiologist made the impression of $\mathrm{CBD}$ stone. The patient was then referred to perform ERCP for a stone removal. 


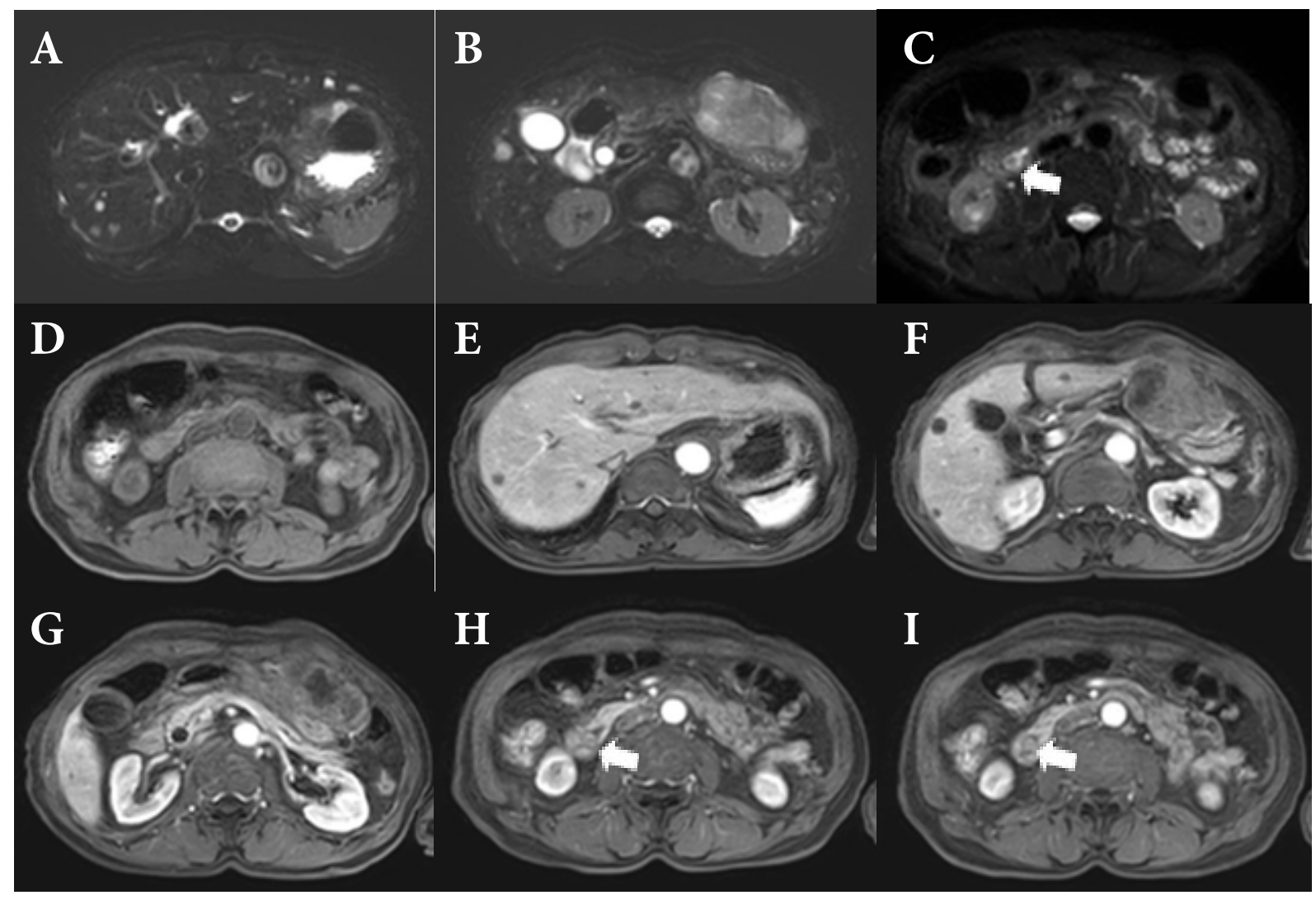

Figure 1. Magnetic Resonance Imaging (MRI) with a hepatocyte specific contrast agent. Axial T2-weighted fat-suppressed fast spin-echo images $(A-C)$ showing moderate dilation of extra- and intrahepatic bile ducts with an oval-shaped intra-luminal filling defect (arrow). Pre (D) and arterial-phase post-contrast axial T1w-3Dfs SGR images (E-I) demonstrating a small smoothly well-defined mildly enhancing nodule in the distal part of a common bile duct (arrow). 
The endoscopic retrograde cholangiography shows a small well-demarcated intraluminal nodule at the distal common bile duct (Figure 2A). The lesion shows no definite stalk, so the stone was diagnosed, and balloon extraction is performed. The soft tissue lesion was accidentally obtained instead of the stone. The real time endoscopy shows a yellowish soft tissue with a lobulated surface (Figure 2B). The tissue was sent for a pathologic evaluation. After the balloon extraction with the presumed total removal of the lesion, a biliary double pigtail stent was inserted in the biliary tract. Three months later, ERCP was repeated, and the stent was removed. Complete occlusion cholangiogram was done in order to expand the CBD lumen for good visualization, which confirmed that there was no recurrence of the tumor.

Tissue pathology shows bile duct adenoma, a tubular pattern. It comprises groups of glands lined with cuboidal cells, possessing low-grade dysplastic nuclei and eosinophilic cytoplasm without an obvious high-grade dysplastic change or malignant transformation. Invasion is not identified (Figure 3).
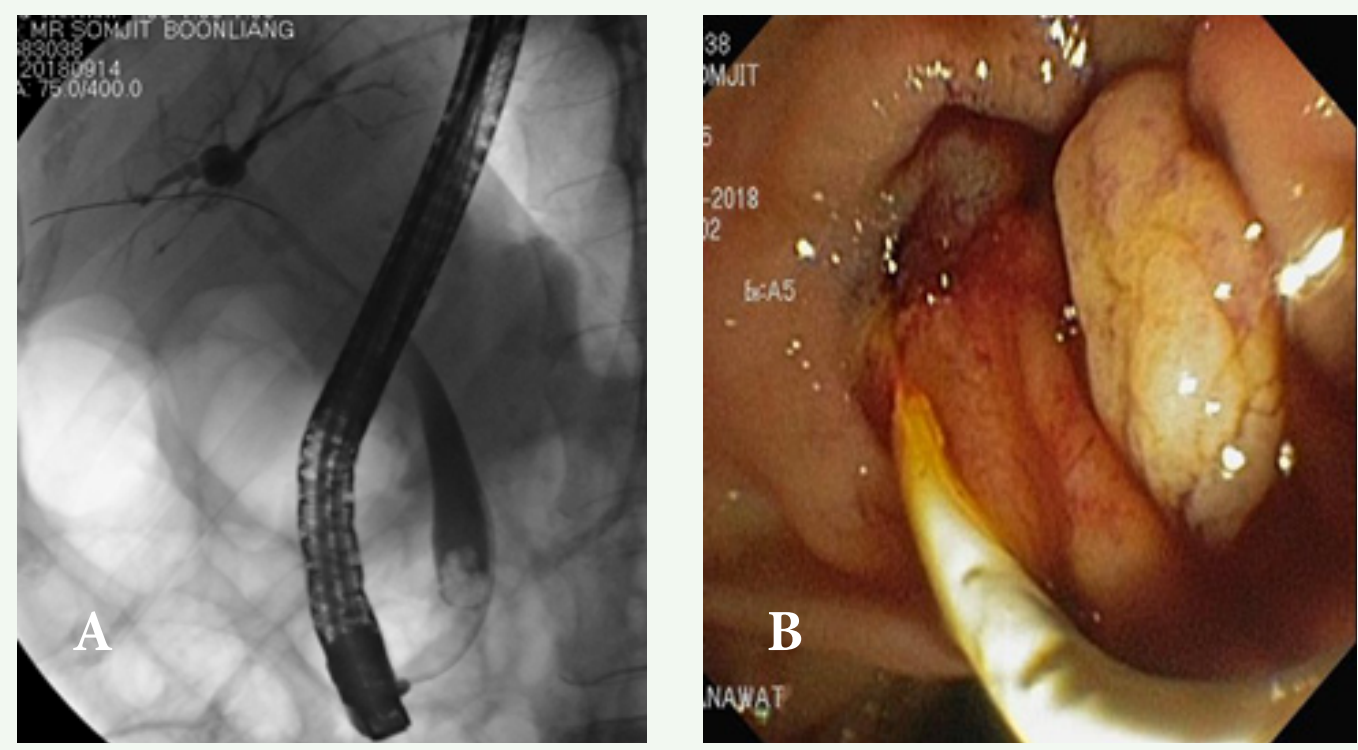

Figure 2. Endoscopic retrograde cholangiography $(A)$ shows a small well-demarcated intraluminal nodule in the distal part of the common bile duct. A stone was diagnosed. Endoscopic photograph (B) at ampulla exhibits that the extracted nodule from the distal part of the common bile duct was a yellowish soft tissue and a smoothly lobulated surface. 

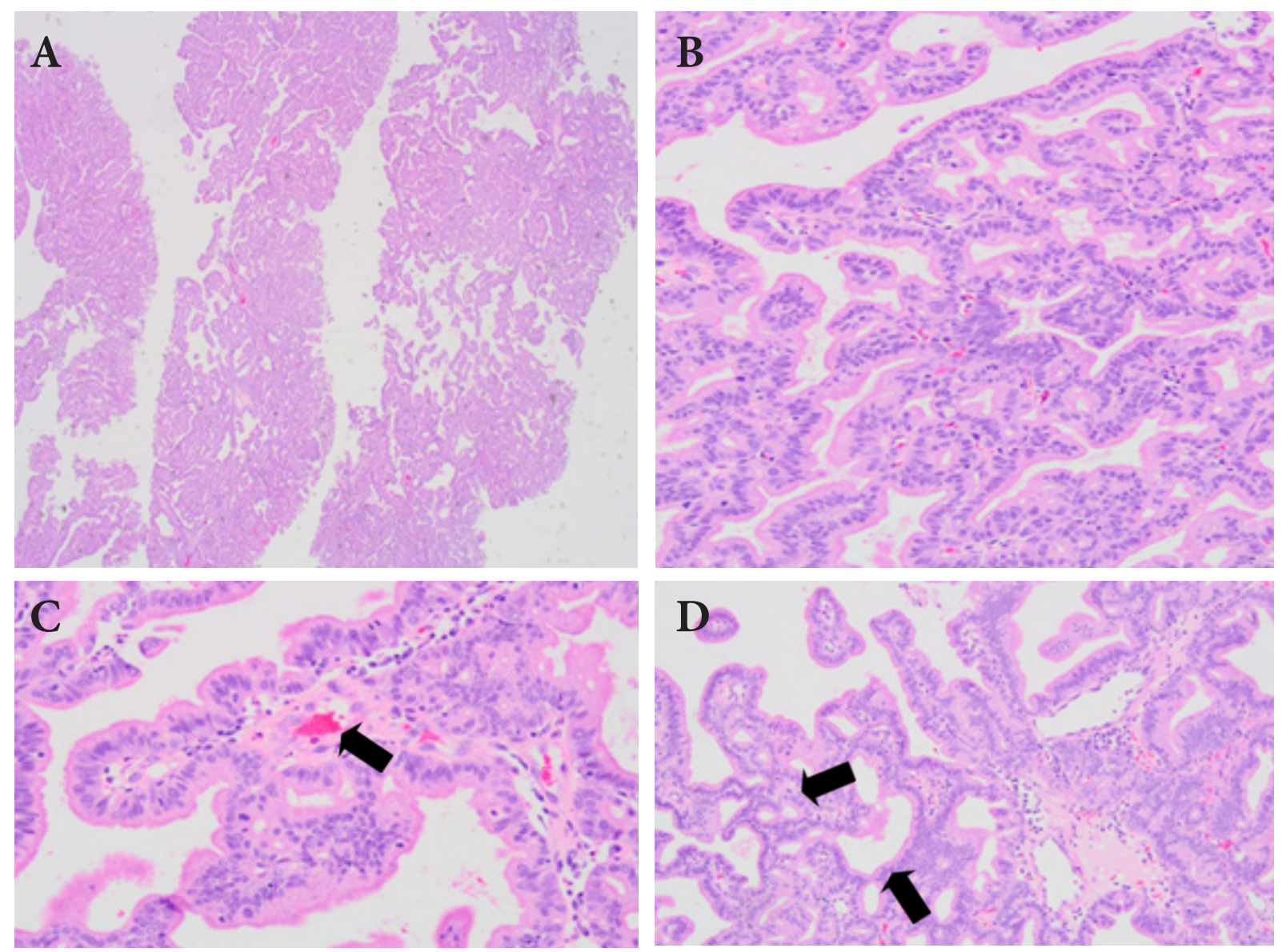

Figure 3. Tissue pathology obtained from a distal common bile duct. Low power field microscopy (A) shows a polypoid lesion High power filed (HPF) (B) image shows a mixed tubulopapillary pattern with cuboidal cell linings, morphologically similar to gallbladder epithelium. HPF image (C) shows a papillary structure with a fibrovascular core (arrow). HPF image (D) shows a complex tubular pattern (arrow). 


\section{Discussion}

Adenoma is a benign tumor of glandular tissue. Gastrointestinal adenoma is usually located in the colon, the rectum, and less frequently, the small bowel[1]. All benign extrahepatic bile duct tumors, including adenoma, are rare[2]. Biliary adenoma are usually found in a surgically removed gallbladder, but can occur in the extrahepatic biliary tree[3]. The World Health Organization Classification of common bile duct adenomas includes tubular adenoma, papillary and tubulopapillary adenoma, biliary cystadenoma, and papillomatosis (or adenomatosis)[1].

Clinical presentations of extrahepatic biliary adenoma are obstructive jaundice, right upper quadrant abdominal pain (biliary colic), ascending cholangitis, biliary pancreatitis and an abnormal liver function test[2]. Cholangiography usually demonstrates adenomas as intraluminal mucosal polypoid lesions similar to gastrointestinal adenoma or polyp. Without demonstration of their stalks, differentiation from a stone is difficult. Densities on computed tomography, signals on Magnetic Resonance Imaging and internal enhancement on either computed tomography or Magnetic Resonance Imaging could confirm their neoplastic nature. However, differentiation from the more common malignant tumors such as extrahepatic polypoid cholangiocarcinoma or ampullary adenocarcinoma is difficult[4].

A surgical resection is the first choice of treatment, an endoscopic resection in an inoperable status by snare polypectomy or forceps has been reported $[3,5]$. There are no reports of the use of an ablative therapy with radiofrequency ablation. There is limited understanding of the malignant potential of biliary adenomas, and no definite guideline for management. However, malignant transformation has also been reported[6]. So prior studies recommended aggressive surgical intervention and close postoperative surveillance when diagnosis of an extrahepatic biliary adenoma[3, 6]. 
In our case, we used balloon extraction and accidentally removed the tumor. We decided to follow up the patient without a surgical resection but also showed no recurrence within 3 months. A few prior studies also show no recurrence after the local resection, but all reports had a follow-up period of less than 1-2 years[6,7].

\section{Conclusion}

Common bile duct adenoma is a rare benign tumor of the extrahepatic biliary tree. Both clinical presentation and imaging finding have limited potential to rule out malignancy. A pathological report is essential for definite diagnosis. Due to report of malignant transformation, surgical resection is the first choice of treatment. However, there were reported cases of endoscopic resection as well as balloon extraction in our report which showed no local recurrence.

Acknowledgement: This case is one of the cases presented in the clinicradio-pathologic session of Nanthana-Kriangkrai-Chotiwattanaphan (NKC) 13th annual meeting which was held on $2^{\text {nd }}$ May 2019 in Phuket, Thailand. We would like to express our sincere gratitude to the NKC Institute for giving us the opportunity to distribute this interesting case. 


\section{References}

1. Albores-Saavedra J, Scoazec JC, Wittekind C, Sripa B, Menck HR, Soehendra $\mathrm{N}$, et al. Carcinoma of the gallbladder and extrahepatic bile ducts. In: Hamilton SR, Aaltonen LA,editors. World Health Organization classification of tumours: pathology and genetics of tumours of the digestive system. Lyon, France: IARC Press; 2000. p. 204-14.

2. Xu HX, Chen LD. Villous adenoma of extrahepatic bile duct: contrastenhanced sonography findings. J Clin Ultrasound 2008;36:39-41. doi: 10.1002/ jcu.20361.

3. Loh KP, Nautsch D, Mueller J, Desilets D, Mehendiratta V. Adenomas involving the extrahepatic biliary tree are rare but have a aggressive clinical course. Endosc Int Open 2016;4: E112-7. doi: 10.1055/s-0041-107897.

4. Aparajita R, Gomez D, Verbeke CS, Menon KV. Papillary adenoma of the distal common bile duct associated with a synchronous carcinoma of the peri-ampullary duodenum JOP 2008;9:212-5.

5. Pracchayakul V, Aswakul P, Kachintorn U. Incidental removal of distal common bile duct adenoma after plastic stent placement. Endoscopy 2012;44 Suppl 2 UCTN:E11-2. doi: 10.1055/s-0031-1291497.

6. Ariche A, Shelef I, Hilzenrat N, Dreznik Z. Villous adenoma of the common bile duct transforming into a cholangiocarcinoma. Isr Med Assoc J2002;4:114950 .

7. Bahuth JJ, Winkley JH. Benign tumor of the common bile duct. Calif Med 1966;307-9. 